\title{
Host Location of a Pupal Parasitoid in a Tritrophic System Compared to a Model Offering Mechanosensory Cues Only
}

\author{
Journal Article \\ Author(s): \\ Fischer, Sabine; Samietz, Jörg; Dorn, Silvia \\ Publication date: \\ 2004-03 \\ Permanent link: \\ https://doi.org/10.3929/ethz-b-000050605
}

Rights / license:

In Copyright - Non-Commercial Use Permitted

Originally published in:

Journal of Insect Behavior 17(2), https://doi.org/10.1023/B:JOIR.0000028569.76708.40 


\title{
Host Location of a Pupal Parasitoid in a Tritrophic System Compared to a Model Offering Mechanosensory Cues Only
}

\author{
Sabine Fischer, ${ }^{1}$ Jörg Samietz, ${ }^{1}$ and Silvia Dorn ${ }^{1,2}$ \\ Accepted October 7, 2003; revised November 13, 2003
}

Several species of hymenopteran parasitoids are able to locate concealed pupal hosts by vibrational sounding. However, the specific role of this technique of mechanosensory host location has not yet been investigated in a natural, tritrophic system with multiple cues. Here we compared the host location of the pupal parasitoid Xanthopimpla stemmator in a tritrophic system with corn borer pupae in maize stem to the behavior on a paper model offering mechanosensory cues only. In general, the behavioral pattern and the behavioral states exhibited by host-searching female parasitoid were identical in the model and in the tritrophic system, while quantitative aspects differed. Our results demonstrate that vibrational sounding maintains its significance for host location in an environment with multiple cues, and that additional cues may increase responsiveness of females.

KEY WORDS: host location; tritrophic system; vibrational sounding; pupal parasitoid; stemborer; Xanthopimpla stemmator.

\section{INTRODUCTION}

For host location, parasitoids of the nonfeeding and stationary pupal stage of endophytic hosts rely heavily on physical cues (Wäckers et al., 1998; Fischer et al., 2001). Plant- or insect-derived contact chemicals are expected to be

${ }^{1}$ Institute of Plant Sciences, Applied Entomology, Swiss Federal Institute of Technology (ETH), Clausiusstrasse 25/NW, CH-8092 Zurich, Switzerland.

${ }^{2}$ To whom correspondence should be addressed. e-mail: silvia.dorn@ipw.agrl.ethz.ch. Fax: +411-6321171. 
less reliable and detectable than they are, for example, for parasitoids of concealed larval hosts (Mattiacci et al., 1999; Dutton et al., 2000). Tactile cues like stemborer tunnel openings (Pfannenstiel et al., 1992), visual cues created by desiccation or discoloration of stems (Fischer et al., 2003), and vibrational cues produced by wriggling pupae (Meyhöfer et al., 1994) guide the parasitoid females to places where host pupae are hidden. Besides responding to these host- or plant-derived physical cues, several species of the hymenopteran families Orussidae and Ichneumonidae have developed a sophisticated host-location strategy. They transmit self-produced vibrations via the antennae onto the substrate covering the host and analyze the reflected signals. This form of echolocation, called vibrational sounding, has so far only been demonstrated for Pimpla instigator (F.) (Henaut and Guerdoux, 1982) and Pimpla turionellae (L.) (Wäckers et al., 1998). However, morphological structures associated with vibrational sounding were found in many species of the subfamily Pimplinae (Broad and Quicke, 2000).

For parasitoid wasps specialized on hosts covered by hard substrate, host location is likely to be particularly time- and energy-consuming. Therefore, highly accurate host-location mechanisms such as vibrational sounding have evolved as an adaptation to the needs of parasitoids of wood-boring, stemboring, soil-dwelling, and cocooned hosts, as well as stem-nesting aculeates (Broad and Quicke, 2000). However, the specific role of vibrational sounding in host location of parasitoids of concealed pupal hosts has not yet been investigated in a natural, tritrophic system with multiple cues.

The endoparasitoid Xanthopimpla stemmator (Thunberg) (Hymenoptera: Ichneumonidae) is specialized on pupae of lepidopteran stemborers (Hailemichael et al., 1994). It can locate a possible host by only using vibrational sounding, indicated by pronounced surface antennation within an area of about $2-3 \mathrm{~cm}^{2}$ above host mimics offering mechanosensory cues only and subsequent ovipositor insertion (Fischer et al., unpublished). To assess the role of vibrational sounding in multisensory host location, we studied the behavior of $X$. stemmator during host location in a tritrophic system consisting of maize plants infested with the European corn borer Ostrinia nubilalis Hübner (Lepidoptera: Pyralidae) and compared it to a paper stem model containing cigarette filters as host mimics. In contrast to the model that offered only mechanosensory cues to the searching parasitoid females, also physical (Smith et al., 1993) and olfactory cues (Turlings et al., 1998) were available on the infested maize in the present study. Besides, the maize plants produced a natural gradient of microclimate characterized by high humidity at the plant (70\% RH) and had a surface texture differing from that of the paper model. If vibrational sounding would play a significant role as a host-location strategy under those more complex stimuli in the tritrophic system, the behavioral pattern and the time spent in characteristic 
behavioral states like antennating should be as prominent as exhibited in the model system with pure mechanosensory cues. The latter was tested in the present study by qualitative and quantitative analysis of the behavior as well as the patterns of transition between behavioral states during host location of X. stemmator.

\section{MATERIALS AND METHODS}

Parasitoid Rearing. The laboratory strain of Xanthopimpla stemmator originated from insects obtained from Marianne Alleyne of the University of Illinois at Urbana-Champaign, USA, in 2000. Subsequently, parasitoids were reared on pupae of the wax moth Galleria mellonella L. Adults were kept in Plexiglas containers $(25 \times 25 \times 25 \mathrm{~cm})$ at $23^{\circ} \mathrm{C}, 70 \% \mathrm{RH}$, during the day and at $15^{\circ} \mathrm{C}, 80 \% \mathrm{RH}$, during the night, with a photoperiod of $16 \mathrm{~L}: 8 \mathrm{D}$. Wasps were fed with honey and water and were allowed to mate. Starting at an age of about 1 month parasitoids were provided with host pupae for oviposition and host-feeding. $X$. stemmator accepted only pupae hidden within a substrate such as paper rolls. After having been exposed to the wasps for 3 to $5 \mathrm{~h}$, parasitized pupae were stored at $24^{\circ} \mathrm{C}, 60 \% \mathrm{RH}$, and a photoperiod of 16L:8D until emergence of the adult wasps, which typically occurred after 3 weeks. Female $X$. stemmator had a life span of up to 4 months.

Maize Cultivation and Infestation. Maize (Zea mays) (hybrid LG 11; Swiss Federal Research Station for Agroecology and Agriculture, ZurichReckenholz), treated with Captolate (50\% Captan, 35\% Antrachinon), was cultivated in a climate chamber at $23^{\circ} \mathrm{C}, 70 \% \mathrm{RH}$, during the day and at $15^{\circ} \mathrm{C}, 80 \% \mathrm{RH}$, during the night, with a photoperiod of 16L:8D. Eggs of Ostrinia nubilalis were obtained from Thomas Meise of the BBA Institute, Darmstadt, Germany. Early-instar larvae feed cryptically in leaf sheaths causing a characteristic "windowing" damage. Final-instar larvae enter the stem and hollow out the internode by their feeding. Pupation normally takes place in the stem in a chamber constructed by the mature larva (Beck, 1987). Single maize plants in the pretasseling stage at an age of 30 days and a stem height of about 70 to $100 \mathrm{~cm}$ were infested with 20 to 30 newly hatched corn borer larvae. The large number of larvae was necessary because of high mortality due to cannibalism between individuals on the same plant. To prevent movement of larvae between different plants, the potted plants were covered individually with gauze bags. Typically, $O$. nubilalis took about 40 days to develop from the time newly hatched larvae were transferred to the plants to the pupal stage in the maize stem. Special attention was paid to avoid any mechanical damage of the maize plants causing additional volatile release. After the experiment, all maize stems into which female parasitoids had inserted their ovipositor during the trial sessions $(n=18)$ were opened 
longitudinally. The number of pupae found per plant ranged from zero in one case (i.e., the larvae infested the plant but died subsequently) up to seven.

Model Setup. Paper cylinders containing a solid section imitating a host pupa are ideal experimental models that were used in a number of studies on host location in Pimpla and Xanthopimpla species (e.g., Hailemichael et al., 1994; Wäckers et al., 1998; Otten et al., 2001). Due to the similarity with the stem- or leaf-roll shelters of their hosts, hollow rounded substrates evoke searching and oviposition behavior in many Ichneumonidae. Our model consisted of two cigarette filters $(1.5 \times 0.8 \mathrm{~cm}$; Gizeh, Germany) placed inside a hollow cylinder $(59 \times 0.8 \mathrm{~cm})$ made of airmail paper (ELCO Atlantic Clipper, Switzerland; $45 \mathrm{~g} \mathrm{~m}^{-2}$ ). The two filters were separated by a distance of $28 \mathrm{~cm}$ and the distance from a filter to the nearest end of the paper roll was $14 \mathrm{~cm}$. These solid sections imitated host pupae within a plant stem and offered the density differences needed by the parasitoids in mechanosensory host location (Wäckers et al., 1998). Since the host mimics could not be detected visually or chemically from the outside, directed orientation can be attributed to vibrational sounding.

Testing Procedures. Trials with maize were performed after pupation of the corn borer. A single infested maize plant was placed in a Plexiglas container $(50 \times 50 \times 150 \mathrm{~cm})$. One female parasitoid was released into the cage and given $30 \mathrm{~min}$ to touch the plant. Starting upon contact of the parasitoid with the plant, a recording of five different behavioral states (defined below) with the software The Observer 3.0 (Noldus, 1995) was made for a duration of $20 \mathrm{~min}$. Each individual parasitoid was used only once. The maize plant was replaced after the experiment if an ovipositor insertion had occured.

In the trials with the model, a single paper cylinder was secured vertically in the center of a Plexiglas container $(60 \times 60 \times 60 \mathrm{~cm})$. It is known that $X$. stemmator prefers vertically oriented stems or stem-like structures. This is probably an adaptation to the cryptic microhabitats in grass communities occupied by the majority of hosts recorded for this species (Hailemichael et al., 1994). All other experimental conditions correspond with the ones described above for the trials on maize. Experiments on both model and maize were performed at a mean temperature of $25^{\circ} \mathrm{C}$ and a mean relative humidity of $40 \%$. In the vicinity of the plant the relative humidity increased to about $70 \%$. The female wasps used in the experiments were 1.5 to 2.5 months old. Their body weight in the maize trials was $39 \pm 4 \mathrm{mg}$ (mean $\pm \mathrm{SD}$ ); in the model trials, $38 \pm 5 \mathrm{mg}$. Individuals were provided with food ad libitum and had previous oviposition experience with Galleria pupae wrapped in cigarette paper (Gizeh Brookfield, Germany; $13 \mathrm{~g} \mathrm{~m}^{-2}$ ).

Definition of Behavioral States. We defined five behavioral states displayed by X. stemmator females during host location on infested maize and 
models, as follows:

Walking: Rapid displacement on the surface of plant or model.

Antennation: Arrestment of the searching individual within an area of about $2-3 \mathrm{~cm}^{2}$ with pronounced surface antennation. Vibrational sounding is indicated by this behavioral state, during which the females transmit pulses via the antennae and receive reflected signals through subgenual organs in their tibiae (Henaut and Guerdoux, 1982; Otten et al., 2001).

Probing: Female stands still and bends the abdomen to place the ovipositor tip on the substrate surface.

Insertion: Ovipositor is inserted into the stem or model. During oviposition females usually fanned their wings in slow or high speed as described for Xanthopimpla punctata (F.) (Pillai and Ramachandran Nair, 1989).

Pausing: Cleaning behavior or no obvious movement at all.

Besides these behavioral states, we recorded the time that a female was absent from the plant or model during the observation period of $20 \mathrm{~min}$.

Data Analysis. Of a total of 32 females that landed on infested maize, 18 behavioral sequences with ovipositor insertion were recorded. From 44 female wasps that landed on the model, 10 recordings with ovipositor insertion were made. The general responsiveness of the wasps, i.e., the number of individuals inserting their ovipositor versus the inactive ones, was analyzed for significant differences between infested maize and the models using a $\chi^{2}$ test. The effect of female body weight on the responsiveness was tested for trials with models and trials with maize using unpaired $t$-tests.

All following analyses were restricted to recordings with ovipositor insertion, as this is the behavioral response indicating host location (Meyhöfer et al., 1997). Transition frequencies from one behavioral state (criterion event) to another (target event) were obtained using a state-lag sequential analysis. Transition probabilities were calculated as the transition frequency from one behavioral state to another divided by the total number of transitions of the parasitoid. The transition probabilities from walking to antennation, from antennation to probing, and from probing to insertion were compared between model and maize trials using Fisher's exact probability test.

The total time spent in each of the five behavioral states was compared between model and maize trials using unpaired $t$-tests. The effect of the number of pupae (factor) on the total duration of each behavioral state in trials with infested maize was tested by a one-way analysis of variance (ANOVA). One recording with ovipositor insertion into a maize stem without pupa was omitted from the analysis. 


\section{RESULTS}

The responsiveness of Xanthopimpla stemmator females, i.e., the proportion of individuals that responded with ovipositor insertion versus those that did not respond, differed significantly between the trials in the model and those in the tritrophic system $\left(\chi^{2}\right.$ test: $\chi^{2}=8.95$, $\left.\mathrm{df}=1, P=0.003\right)$. More females inserted the ovipositor into maize plants infested with Ostrinia nubilalis pupae $(56 \%)$ than into paper cylinders with a cigarette filter as a host mimic $(23 \%)$. Body weight of the females did not significantly affect their responsiveness (unpaired $t$-test; model, $t=1.03$, df $=42, P=0.31$; maize, $t=-0.52, \mathrm{df}=30, P=0.61)$.

Landing of the parasitoids on the maize or the model was followed by walking irrespective of the system tested. Subsequently, the parasitoid females switched mainly to antennation on the maize plant. On the model, most females switched from walking to pausing. The corresponding difference in transition probability from walking to antennation was significant between the infested plants and the model (Table I). Antennating wasps either started to probe with their ovipositors or changed back to walking. Transition probabilities from antennation to probing did not show significant differences between the plant and the model system, amounting to 0.30 and 0.37 , respectively (Table I). After probing, a searching female typically inserted the ovipositor several times into an area of interest. Again, the transition probabilities for the two systems studied were not significantly different, amounting to 0.64 for the maize and 0.63 for the model system (Table I). Repeated ovipositions were normally interrupted by periods of strong antennation.

Of the total observational period, the proportion of time spent without contact to the plant or model amounted to $20 \pm 6 \%$ (mean \pm SE) in the tritrophic system and $25 \pm 7 \%$ in the model trials. Although host-searching females did not show significant differences in time spent probing on the infested maize and on the model (Fig. 1; unpaired $t$-test; $t=0.74, \mathrm{df}=26$,

Table I. Comparison of Transition Probabilities (TP) Between Behavioral States Associated with Mechanosensory Host Location in Xanthopimpla stemmator Females on Maize Plants Infested with Ostrinia nubilalis Pupae and on Paper-Cylinder Models with Cigarette Filters as Host Mimics

\begin{tabular}{lcccccc}
\hline \multirow{2}{*}{\multicolumn{1}{c}{ Transition }} & \multicolumn{2}{c}{ Maize } & & \multicolumn{2}{c}{ Model } & \\
\cline { 2 - 3 } & TP & Counts & & TP & Counts & \\
\hline Walking-antennation & 0.48 & $162 / 340$ & & 0.22 & $40 / 182$ & 0.001 \\
Antennation-probing & 0.30 & $85 / 281$ & & 0.37 & $31 / 83$ & 0.38 \\
Probing-insertion & 0.64 & $72 / 112$ & & 0.63 & $26 / 41$ & 1.00 \\
\hline
\end{tabular}

Note. Counts: absolute transition frequency/total number of transitions to any other behavioral state. 


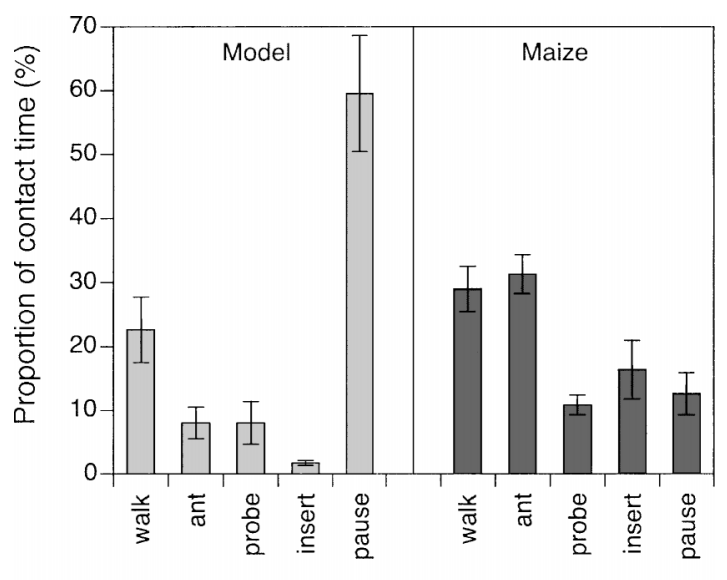

Behavioral states

Fig. 1. Proportion of time (mean \pm SE) spent by Xanthopimpla stemmator in five different behavioral states on a model offering mechanosensory cues only $(n=10)$ and on maize plants with hidden Ostrinia nubilalis pupae offering multiple stimuli $(n=18)$. Durations of behavioral states are given as a percentage of the total time the insect was in contact with the plant or model.

$P=0.47)$, the total duration of antennation and insertion differed significantly between the two systems (Fig. 1; antennation, $t=4.86$, df $=26$, $P<0.001$; insertion, $t=2.22, \mathrm{df}=26, P=0.04)$. The time spent walking was the same on infested maize and on the model, but on the model the individuals spent significantly more time pausing (Fig. 1; walking, $t=1.32$, df $=26, P=0.20$; pausing, $t=4.33$, df $=26, P<0.001)$. The number of pupae in the maize stem had a significant influence on the total duration of antennation but not on the time spent in other behavioral states (one-way ANOVA; antennation, $F=6.3$, df $=4,12, P<0.01$; other behavioral states, not significant at $5 \%$ level). When only trials with one or two stemborer pupae were compared to the model trials with two host mimics, the duration of antennation was still significantly longer on maize (unpaired $t$-test: $t=4.72, \mathrm{df}=19, P<0.001)$. Although $X$. stemmator females spent most of their time on the plant with antennation $(31 \pm 3 \%)($ mean $\pm \mathrm{SE})$, pausing was the most frequent behavior in the model system (60 $\pm 9 \%$ ) (Fig. 1).

\section{DISCUSSION}

In general, the behavioral pattern and the behavioral states exhibited by host-searching female parasitoids were identical in the model and the 
tritrophic system. This finding implies that, as long as mechanosensory cues are provided, Xanthopimpla stemmator females do not change their hostsearching pattern according to additional cues. All qualitative aspects of the mechanosensory host-searching behavior of the parasitoid recorded in the model system are represented in the host-searching behavior in the natural system. This demonstrates that vibrational sounding maintains its significance for host location in an environment with multiple cues.

However, quantitative aspects of the host-searching behavior differed in the model and the tritrophic system. The increased responsiveness on infested maize was likely due to the availability of multiple cues including visual cues derived from the plant surface (Smith et al., 1993; Fischer et al., 2001), olfactory cues such as pupal odor or larval frass (Hailemichael et al., 1994; Turlings et al., 1998), and/or host vibrations (Hailemichael et al., 1994) that revealed the presence of a living host in the tritrophic system. Furthermore, the microclimatic conditions with high humidity and the tactile properties of the stem surface could have enhanced the responsiveness of the searching parasitoids.

Most interestingly, host-searching behavior in the tritrophic system was stimulated in a way that revealed the importance of vibrational sounding as a host-location strategy in a natural, multisensory environment. The duration of the behavioral state antennation, which indicates vibrational sounding activity, and the number of transitions from walking to antennation were even higher in the tritrophic system than in the model system. The lack of visual and chemical cues in the model system may be reflected in the long time the parasitoids spent pausing in this system. If we had used a living pupa instead of a cigarette filter in the model system, it is likely that the additional host-derived cues would have increased the time spent for antennation, probing, and insertion by the female parasitoids. However, the transition probabilities from antennation to probing and then to ovipositor insertion were not significantly different between the model and the tritrophic system. This suggests that the behavioral sequence initiated by vibrational sounding is predetermined and that, once the substrate has been examined by antennation, probing and insertion are following with high probabilities.

In order to pinpoint the proper location for an ovipositor insertion, parasitoids of concealed pupae depend strongly on physical cues. These cues may interact in a similar manner as vibrational and visual cues do in host location of Pimpla turionellae (Fischer et al., 2001) and they may also be used in a hierarchical way along with olfactory stimuli as described for X. stemmator (Hailemichael et al., 1994). Our findings indicate that vibrational sounding is a significant strategy for location of the endophytic pupal host in this parasitoid and that its role is not diminished in multisensory host location. 


\section{ACKNOWLEDGMENTS}

We are grateful to Kathrin Tschudi-Rein and an anonymous referee for valuable comments on early drafts of the paper. We thank Patrick Bussmann and Daniel Trujillo Villegas for help with insect rearing and Tanja Christoffel for plant growing.

\section{REFERENCES}

Beck, S. D. (1987). Developmental and seasonal biology of Ostrinia nubilalis. Agr. Zool. Rev. 2: 59-96.

Broad, G. R., and Quicke, D. L. J. (2000). The adaptive significance of host location by vibrational sounding in parasitoid wasps. Proc. Roy. Soc. Lond. B Biol. 267: 2403-2409.

Dutton, A., Mattiacci, L., and Dorn, S. (2000). Plant-derived semiochemicals as contact host location stimuli for a parasitoid of leafminers. J. Chem. Ecol. 26: 2259-2273.

Fischer, S., Samietz, J., Wäckers, F. L., and Dorn, S. (2001). Interaction of vibrational and visual cues in parasitoid host location. J. Comp. Physiol. A 187: 785-791.

Fischer, S., Samietz, J., Wäckers, F. L., and Dorn, S. (2003). Perception of achromatic cues during host location of a pupal parasitoid. Entomol. Exp. Appl. 106: 63-66.

Hailemichael, Y., Smith, J. W., Jr., and Wiedenmann, R. N. (1994). Host-finding behaviour, host acceptance, and host suitability of the parasite Xanthopimpla stemmator. Entomol. Exp. Appl. 71: $155-166$.

Henaut, A., and Guerdoux, J. (1982). Location of a lure by the drumming insect Pimpla instigator (Hymenoptera, Ichneumonidae). Experientia 38: 346-347.

Mattiacci, L., Hütter, E., and Dorn, S. (1999). Host location of Hyssopus pallidus, a larval parasitoid of the codling moth, Cydia pomonella. Biol. Control 15: 241-251.

Meyhöfer, R., Casas, J., and Dorn, S. (1994). Host location by a parasitoid using leafminer vibrations: characterizing the vibrational signals produced by the leafmining host. Physiol. Entomol. 19: 349-359.

Meyhöfer, R., Casas, J., and Dorn, S. (1997). Vibration-mediated interactions in a host-parasitoid system. Proc. Roy. Soc. Lond. B Biol. 264: 261-266.

Otten, H., Wäckers, F., Battini, M., and Dorn, S. (2001). Efficiency of vibrational sounding in the parasitoid Pimpla turionellae is affected by female size. Anim. Behav. 61: 671-677.

Pfannenstiel, R. S., Browning, H. W., and Smith, J. W., Jr. (1992). Searching behavior of Pediobius furvus (Hymenoptera: Eulophidae) for Eoreuma loftini (Lepidoptera: Pyralidae) in sugarcane. J. Econ. Entomol. 85: 384-388.

Pillai, G. B., and Ramachandran Nair, K. (1989). Observations on Xanthopimpla punctata F. (Hymenoptera: Ichneumonidae), a pupal parasitoid of Opisina arenosella Wlk. J. Plant. Crops 16: 173-177.

Smith, J. W., Jr., Wiedenmann, R. N., and Overholt, W. A. (1993). Parasites of Lepidopteran Stemborers of Tropical Gramineous Plants, ICIPE Science Press, Nairobi.

Turlings, T. C. J., Bernasconi, M., Bertossa, R., Bigler, F., Caloz, G., and Dorn, S. (1998). The induction of volatile emissions in maize by three herbivore species with different feeding habits: Possible consequences for their natural enemies. Biol. Control 11: 122-129.

Wäckers, F. L., Mitter, E., and Dorn, S. (1998). Vibrational sounding by the pupal parasitoid Pimpla (Coccygomimus) turionellae: An additional solution to the reliability-detectability problem. Biol. Control 11: 141-146. 